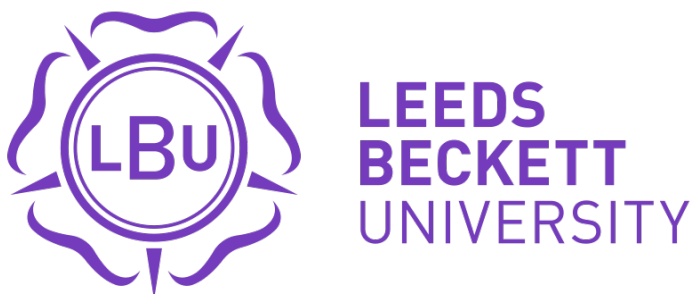

Citation:

Bazarhanova, A and Kor, A and Pattinson, C (2017) A Belief Rule-Based Environmental Responsibility Assessment for Small and Medium-Sized Enterprises. IEEE Xplore. DOI: https://doi.org/10.1109/FTC.2016.7821673

Link to Leeds Beckett Repository record:

https://eprints.leedsbeckett.ac.uk/id/eprint/3349/

Document Version:

Article (Accepted Version)

The aim of the Leeds Beckett Repository is to provide open access to our research, as required by funder policies and permitted by publishers and copyright law.

The Leeds Beckett repository holds a wide range of publications, each of which has been checked for copyright and the relevant embargo period has been applied by the Research Services team.

We operate on a standard take-down policy. If you are the author or publisher of an output and you would like it removed from the repository, please contact us and we will investigate on a case-by-case basis.

Each thesis in the repository has been cleared where necessary by the author for third party copyright. If you would like a thesis to be removed from the repository or believe there is an issue with copyright, please contact us on openaccess@leedsbeckett.ac.uk and we will investigate on a case-by-case basis. 


\title{
A Belief Rule-Based Environmental Responsibility Assessment for Small and Medium-Sized Enterprises
}

\author{
A. Bazarhanova, Dr. Ah-Lian Kor, and Prof. Colin Pattinson
}

\begin{abstract}
This research proposes the use of a Belief Rule-Based approach to assess an enterprise's level commitment to environmental issues. Participating companies will have to complete a structured questionnaire. An automated analysis of their responses will determine their environmental responsibility level. This is followed by a recommendation on how to progress to the next level. The recommended best practices will help promote understanding, increase awareness, and make the organization greener. BRB systems consist of two parts: Knowledge Base and Inference Engine, which are used to derive valid conclusions from rules, established by experts with domain-specific knowledge. The knowledge base in this research is constructed after an in-depth literature review, critical analyses of existing environmental performance assessment models and primarily guided by the EU Draft Background Report for the development of an EMAS Sectoral Reference Document on "Best Environmental Management Practice in the Telecommunications and ICT Services Sector". The reasoning algorithm of a selected Drools JBoss BRB inference engine is forward chaining. However, the forward chaining mechanism is not equipped with uncertainty handling. Therefore, a decision is made to deploy an evidential reasoning and forward chaining with a hybrid knowledge representation inference scheme to accommodate imprecision, ambiguity and fuzzy types of uncertainties. It is believed that such a system generates well balanced, sensible and Green ICT readiness adapted results, to help enterprises focus on making improvements on more sustainable business operations.
\end{abstract}

Keywords - Belief Rule-Based, Green ICT, SMEs assessment, Environmental Responsibility, Environmental Impact Assessment, Sustainability, Knowledge Based System, Reasoning with Uncertainty.

\section{INTRODUCTION}

Companies nowadays, seem to show an increasing commitment to more sustainable behaviour. Environmental responsibility is one of the pillars of a broader Corporate Social Responsibility term. The European Commission has previously defined Corporate Social Responsibility (CSR) as "a concept whereby companies integrate social and environmental concerns in their business operations and in their interaction with their stakeholders on a voluntary basis" [1]. Starting from the early 90s, due to legislation and community pressure, companies began to initiate environmental management campaigns.

Recommendations in Smart 2020 report [2] promote ICT infrastructure deployment to mitigate the pollution, waste, energy usage, etc. Undoubtedly, the ICT sector offers many applications that can bring numerous positive impacts for the natural environment. Some of them are: information, digitization, transport dematerialization, or warehouse and office space reduction [3]. The environmental impacts of ICT largely depend on how ICT applications perform and also human energy consumption behaviour.

Due to legislation pressure and increase of community awareness, a variety of environmental management systems, standards and tools are being developed and used in order to assist companies to become more environmental friendly. Each of them has its own particular benefits and advantages, but there is no indication of which of them is better for the company's current state. The primary focus of an enterprise's environmental management depends on which industrial sector it is in. Companies might take a proactive approach to

A. Bazarhanova is an Erasmus Mundus Master's Program student in Pervasive Computing \& Communications for sustainable Development (PERCCOM), Leeds Beckett University, Leeds LS6 3QS, U.K. (e-mail: bazarhanova@ gmail.com).

Dr. Ah-Lian Kor (PhD, MBCS), Senior Lecturer, Course Leader for MSc Sustainable Computing School of Computing, Creative Technologies and Engineering, Faculty of Arts, Engineering, and Technology Leeds Beckett University, Leeds LS6 3QS, U.K. (e-mail: a.kor@leedsbeckett.ac.uk).

Professor Colin Pattinson, BSc (Hons), PhD, MBCS, Head of School of Computing, Creative Technologies and Engineering, Faculty of Arts, Environment and Technology, Leeds Beckett University, Leeds LS6 3QS, U.K. (e-mail: c.pattinson@ leedsbeckett.ac.uk). 
implementing environmental practices based on specific ISO standards relevant to their industry in order to reduce the environmental impact of their activities. Nevertheless, this paper concentrates on a more generic and aggregated perspective of defining the environmental responsibility of a company.

How to measure the Environmental Responsibility level of SMEs? Which is the recommending path that companies should follow towards environmental performance excellence? This research addresses these questions. Therefore, the research aim primarily focuses on the development of a novel assessment and decision support model to help companies evaluate their current state followed by recommendations of behavioural and operational best practices to enhance their environmental responsibility level. This paper demonstrates the feasibility of Belief RuleBased (BRB) approach in the assessment of enterprise's level commitment to environmental issues.

The remaining of the paper is structured as follows. Section two presents the existing corporate environmental impact assessment models. Section three provides an overview the theoretical basis of Belief Rule-Based methodology applied in research. Section four presents the architecture and implementation of the proposed Environmental Responsibility (ER) BRB assessment system. BRB inference validation and performances comparison with a Fuzzy Inference Systems (FIS) theory are then presented. A final section concludes the paper with findings, limitations of this research, as well as potential avenues for future research.

\section{LITERATURE REVIEW}

Various evaluation approaches and models for the assessment of companies' environmental impact have evolved. Some of most well-known are SURF Green ICT Maturity Model [4], Sustainability Maturity Model from Industrial Research Institute [5], Sustainability Management Maturity Model of FairRidge Group, Systematic action plan from Fachgruppe Green IT [6], UK HM Government Green ICT Maturity Model [7], Green IT Readiness Framework [8] and SustainaBits Framework and Rating System for Sustainable IT [9] by using which organizations may benefit in raising environmental issues awareness.

Works dedicated to data centers assessment and greening operations have been intentionally excluded from the review. Most of the models surveyed are research related models which require minimum knowledge on Green ICT domain and are in formats of scientific works, tables and publications or are abstract and conceptual [8], [9] mitigating the chances to be adopted by non-academic organizations. Some models [4], [5] include an actual assessment by assigning scores per categories, but are not applicable for small and medium-sized enterprises. Most of the models focus on eliminating negative impacts of ICT infrastructure, whereas SMEs need a simple, comprehensive, easy to use and access tool for an assessment of their level of environmental responsibility.

It is evident from literature review that Green ICT and ICT for Greening domain fundamentals in a corporate context need a proper classification and standardization, recognized both by industry and academia. Categorization inconsistencies are demonstrated in models above, and expected to be even more diversified among those which were not identified, skipped or missed. Also, assessment systems miss qualitative reviews and adaptations towards targeted user groups [6], [7], [8] Environmental responsibility level assessment is a multidimensional, observational process that requires a more rigorous reasoning approach to handle uncertainties, imprecisions and at the same time, be positive perspective oriented.

Environmentally Responsibility assessment is characterized by a number of identified factors which are qualitative in nature and can be assessed based on human judgment. Thus, a general ER assessment problem for SMEs could be addressed without a detailed and rigorous audit conducted by affiliated authorities. Such an approach would be able to handle uncertainties, vagueness and fuzziness. Assessment models presented above follow mostly traditional approaches in Green readiness assessment and reasoning, which are incapable of producing accurate ER level results. Expert systems are widely used to deal with knowledge-based decision support systems. Thus, the Belief Rule-Based approach with its ability to infer uncertain knowledge in the domain of Environmentally Responsibility has been applied in this research.

\section{RESEARCH METHODOLOGY}

\section{A. BRB inference reasoning}

Belief Rule-Based Expert Systems consist of two parts: Knowledge Base and Inference Engine, which are used to derive conclusions from rules, either established by experts with domain-specific knowledge, historical data or observation facts provided by users. An Inference Engine is a core algorithm of a BRB system and the following section will examine available reasoning patterns to justify the selection of forward and backward chaining inference 
for the rule-based engine.

First step in building knowledge base of a BRB system is to identify relevant 1) antecedent attributes, 2) types of uncertainties and 3) corresponding weights, which are then form a generic domain knowledge representation scheme using a belief structure [10].

Belief rule-based schema is defined as follows:

$$
\begin{gathered}
\operatorname{IF} x_{1} \text { is } A_{1}^{k} \wedge x_{2} \text { is } A_{2}^{k} \wedge \ldots \wedge x_{T k} \text { is } A_{T k}^{k}, \\
\operatorname{THEN}\left\{\left(D_{1}, \beta_{1, k}\right),\left(D_{2}, \beta_{2, k}\right) \ldots\left(D_{n}, \beta_{n, k}\right),\right\} \\
\text { where } \sum_{n=1}^{N} \beta_{n, k} \leq 1
\end{gathered}
$$

with a rule weight $\theta_{k}$ and attribute weight $\delta_{1, k}, \delta_{2, k} \ldots \delta_{T k, k}, k \in\{1 \ldots L\}$.

Here, $x_{1}, x_{2} \ldots x_{1 T k}$ denote the antecedent variables in the $k_{t h}$ rule. These attributes belong to the set of antecedent variables $X=\left\{x_{i} ; i=1 \ldots T\right\}$ in which each element takes a value from an array of finite sets $A=$ $\left\{A_{1} \ldots A_{t}\right\}$. The vector $A_{i}=\left\{A_{i, n}: n=1 \ldots N_{i}=\left|A_{i}\right|\right\}$ is defined as the set of referential attributes for antecedent variable $x_{i}$. In the $k_{t h}$ rule, $A_{i}^{k}$ represents the referential value corresponding to $i_{t h}$ antecedent variable. $T_{k}$ denotes the total number of antecedent attributes in the $k_{t h}$ rule; $\beta_{n, k}$ is a belief degree to which $D_{n}$ is assumed to be consequent, taking into account the logical relationship of the $k_{t h}$ rule:

Fk: $x_{1}$ is $A_{1}^{k} \wedge x_{2}$ is $A_{2}^{k} \wedge \ldots \wedge x_{T k}$ is $A_{T k}^{k}$

If $\sum_{n=1}^{N} \beta_{n, k}=1$ the $k_{t h}$ rule is said to be complete and incomplete otherwise [10].

For example in the case for ER assessment:

$$
\begin{gathered}
R_{k}: \text { if the use of ecolabelled equipment is high and switch-off and standby policy is } \\
\text { medium and standards compliant strategy adoption is high\}, } \\
\text { then ER level is }\{(\text { good, 0.7), (average, 0.2), (fair, 0.1), (poor, 0)\}, }
\end{gathered}
$$

where belief distribution representation for ER is considered good with $70 \%$ confidence, $20 \%$ for average and $10 \%$ sure that ER level is fair. In this belief rule, the total degree is $0.7+0.2+0.1=1$, so that the assessment is complete [10]. The total degree $\alpha_{k}$ with input match of $A^{k}$ antecedent in the $k_{t h}$ rule is calculated by:

$$
\alpha_{k}=\varphi\left(\left(\delta_{k 1}, \alpha_{k 1}\right) \ldots\left(\delta_{k T_{k}}, \alpha_{T k}^{k}\right)\right)
$$

where $\varphi$ is an aggregation function for $T_{k}$ antecedents in $k_{t h}$ rule and $\delta_{k_{1}}(i=1 \ldots T k)$ is the weight of the $i_{t h}$ antecedent variable. An aggregation function for subjective probabilities generation is " $\wedge$ " operator, i.e $\varphi_{\text {sum }}(a, b)=a+b-a b$ [11]. Particularly, the consequent part of a rule is true, if only all antecedent variable meet rule conditions, so following weighted multiplicative aggregation function was used:

$$
\begin{gathered}
\alpha_{k}=\prod_{i-1}^{T_{k}}\left(\alpha_{i}^{k}\right)^{\overline{\delta_{k l}}} \\
\text { where } \overline{\delta_{k l}}=\frac{\delta_{k i}}{\max _{i=1 \ldots T k}\left\{\delta_{k i}\right\}}
\end{gathered}
$$

Assuming that $u\left(D_{j}\right)$ is the utility of an individual consequent variable, single value converted result is equal to:

$$
u\left(S\left(A^{*}\right)\right)=\sum_{j=1}^{N} u\left(D_{j}\right) \beta_{j}
$$

The overall belief degrees are measured by individual antecedent degrees of the $k_{t h}$ rule activated by an input which is a building base for the overall output belief degree.

\section{B. Knowledge Base in ER}

As it is mentioned earlier, knowledge base in belief rule-based systems is either established by experts with domain-specific knowledge, historical data or observation facts or statistics. In this research, it is based on an indepth literature review and validations of experts in Green. Here, $V_{j}$ denotes the category, e.g. V1 - Equipment procurement compliant with Green ICT guidelines and the optimization of enterprise operations.

TABLE $1-K B$ structure 
V1: Equipment procurement compliance with Green ICT guidelines

V2: Energy performance improvement and monitoring V3: Energy aware networks engineering adherence V4:Social commitment V5:Waste management
$A_{1}^{1}, A_{2}^{1}, A_{3,}^{1} A_{4,}^{1}, A_{5}^{1}$

$A_{1}^{2}, A_{2}^{2}, A_{3,}^{2} A_{4}^{2}$

$$
A_{1}^{3}, A_{2,}^{3} A_{3}^{3}
$$

$A_{1}^{4}, A_{2,}^{4}, A_{3,}^{4}, A_{4}^{4}$

$A_{1}^{5}, A_{2}^{5}$

Knowledge base consists of $5 V_{j}$ parent categories and $17 A_{j}^{i}$ antecedent attributes. Each category consists of antecedent attributes that compose a set of questionnaire items that the user will need to answer. In order to provide mathematical handling of various input data types and uncertainties handling, a set of available referential values is described as $\{(\mathrm{High}, 0.0)$, (Medium, 0.0), (Low, 0.0)\}. It is important to mention that the research has followed several iterations in refining knowledge base categorization: initially there were 8 independent parent categories, which were leading to $3^{\wedge} 8=6561$ cases of combinations to consider, adding additional complications and overhead. That has been later merged into 5 categories for simplicity and integrity purposes. The total ER index is calculated (1), by aggregating $\mathrm{N}=5$ number of parent categories, which in turn consists of $\sum_{i=1}^{N} A_{j}^{i}$ aggregation of corresponding antecedents. $A_{j}^{i}$ represents corresponding questionnaire item for each $V_{j}$ category.

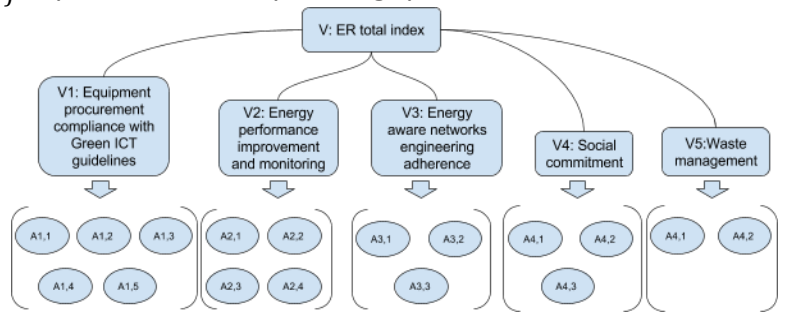

Fig. 1. Knowledge base tree

Having 5 antecedent parent categories with 3 referential values 243 total number of rules was obtained. A total number of 243 rules is determined based on the number of categories

$X=\left\{x_{i} ; i=1 \ldots T\right\}$, where $T=5$ and 3 referential attributed (high, medium, low): $3^{5}=243$. To enumerate all possible combinations $R$ language for statistical computing and graphics was used (function expand.grid(1:3,1:3,1:3,1:3,1:3). Below is the extract of a matrix with 243 rules of inference:

TABLE 2 - Rule base matrix

\begin{tabular}{|c|c|c|c|}
\hline Rule id & $\begin{array}{l}\text { Rule } \\
\text { weight }\end{array}$ & IF & THEN \\
\hline 1 & 1 & $\begin{array}{l}V_{1} \text { is } H \& V_{2} \text { is } H \& V_{3} \text { is } H \& V_{4} \text { is } \\
H \& V_{5} \text { is } H\end{array}$ & $\begin{array}{l}V \text { is }\{H\} \text { or } \\
V \text { is }\{(H, 1.0),(M, 0.0),(L, 0.0)\}\end{array}$ \\
\hline 2 & 1 & $\begin{array}{l}V_{1} \text { is } M \& V_{2} \text { is } H \& V_{3} \text { is } H \& V_{4} \text { is } \\
H \& V_{5} \text { is } H\end{array}$ & $\begin{array}{l}V \text { is }\{H\} \text { or } \\
V \text { is }\{(H, 0.9),(M, 0.1),(L, 0.0)\}\end{array}$ \\
\hline 3 & 1 & $\begin{array}{l}V_{1} \text { is } L \& V_{2} \text { is } H \& V_{3} \text { is } H \& V_{4} \text { is } H \\
\& V_{5} \text { is } H\end{array}$ & $\begin{array}{l}V \text { is }\{H\} \text { or } \\
V \text { is }\{(H, 0.8),(M, 0.2),(L, 0.0)\}\end{array}$ \\
\hline 4 & 1 & $\begin{array}{l}V_{1} \text { is } H \& V_{2} \text { is } M \& V_{3} \text { is } H \& V_{4} \text { is } \\
H \& V_{5} \text { is } H\end{array}$ & $\begin{array}{l}V \text { is }\{H\} \text { or } \\
V \text { is }\{(H, 0.8),(M, 0.2),(L, 0.0)\}\end{array}$ \\
\hline
\end{tabular}




$$
\begin{array}{ll}
V_{1} \text { is } L \& V_{2} \text { is } L \& V_{3} \text { is } L \& V_{4} \text { is } L & V \text { is }\{L\} \text { or } \\
\& V_{5} \text { is } L & V \text { is }\{(H, 0.0),(M, 0.0),(L, 1.0)\}
\end{array}
$$

Here, $\{H\}$ is a high, $\{M\}$ is a medium and $\{L\}$ is a low degree of Environmentally Responsibility index. Table 2 presents two different approaches for producing the total index: $E R$ is $\{H\}$ with implicit uncertainty handling and $E R$ is $\{(H, 1.0),(M, 0.0),(L, 0.0)\}$ with explicit uncertainty handling $(1)$. It has been decided to keep the weights to be one for all the rules, i.e. assigning the same importance to each rule. Examples of a belief rule taken from Table 2 are:

R1: IF Energy performance improvement and monitoring is High and Energy performance improvement and monitoring is High and Energy aware networks engineering adherence is High and Social commitment is High and Waste management is High THEN ER index is High;

R2: IF Energy performance improvement and monitoring is Low and Energy performance improvement and monitoring is High and Energy aware networks engineering adherence is Medium and Social commitment is Low and Waste management is Medium THEN ER index is Medium,

R3: IF Energy performance improvement and monitoring is Low and Energy performance improvement and monitoring is High and Energy aware networks engineering adherence is Low and Social commitment is Low and Waste management is Low THEN ER index is Low,

where belief degrees are attached to three referential values and weighted equal. Upon inference completion total ER index $\left(\sum_{i=1}^{N} V_{n}\right)$ is generated with the following breakdown: Initial level for 0-20\% range, Beginning 20-40\%, Improving $40-60 \%$, Succeeding $60-80 \%$ and Leading $80-100 \%$ accordingly. The total ER index is displayed without uncertainties in a single deterministic value in percentages, i.e. $V$ is $\{H\}$ or $V$ is $\{M\}$ or $V$ is $\{L\}$. Additionally, output result shows the total ER index score and sub-category score breakdown in \%.

\section{ER ASSESSMENT SYSTEM}

As a proof of concept, a Java web-application "Environmental Responsibility Toolkit for SMEs" has been developed, with JBoss Drools inference engine to provide the reasoning mechanism. The inference engine has been populated with rules described in Table 2, where an inference starts iteratively searching for the pattern-match of an input and if-then clause. If it is true, the relevant then clause is fired triggering an appropriate action.

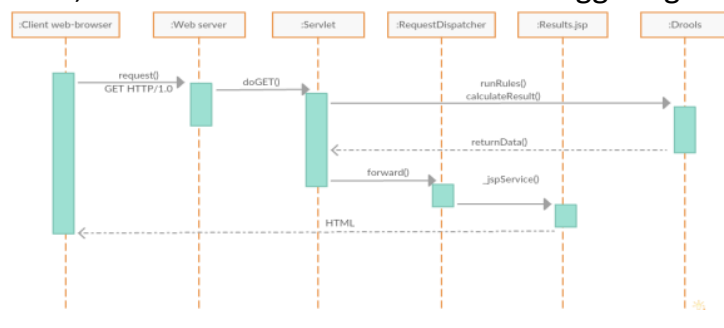

Fig. 2. Web-based ER assessment sequence diagram

The assessment itself encompasses a questionnaire with 17 items (Fig. 3) to be responded with predefined degrees of uncertainty for each rule defined in Table 2.

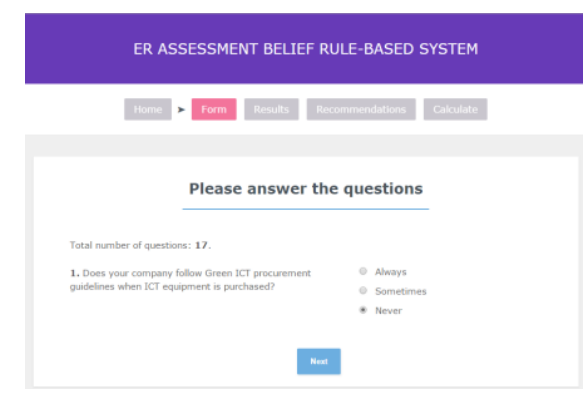

Fig. 3. User input questionnaire 
Upon completion of the questionnaire, the Results is automatically analyzed and the page is displayed with a total ER index score and sub-category score breakdown (Fig. 4).

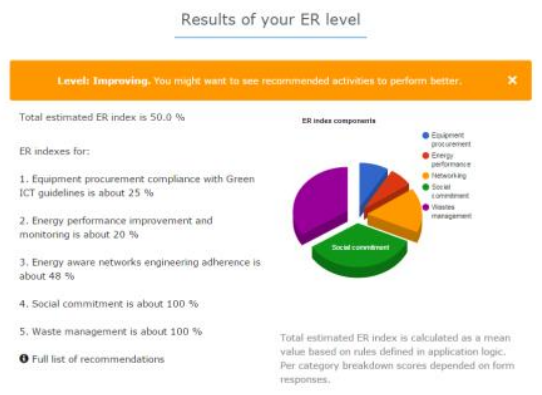

Fig. 4. ER index results

Also, based on the results, an individualized set of recommendations to improve the Environmentally Responsibility level is presented.

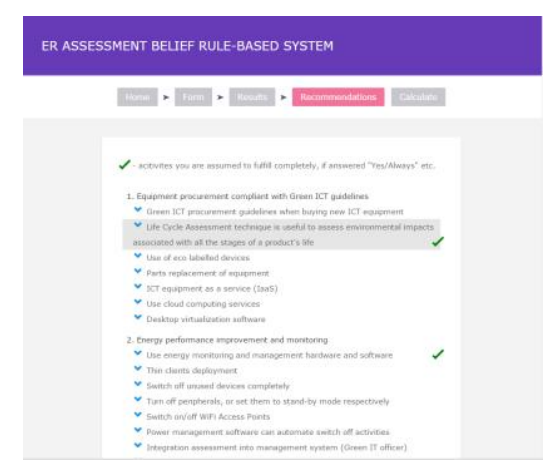

Fig. 5. Recommendations section

Recommendations are based on EU Draft Background Report for the development of an EMAS Sectoral Reference Document "Best Environmental Management Practice in the Telecommunications and ICT Services Sector" [12].

\section{BRB AND FIS PERFORMANCE}

This section presents validation results of BRB system developed with a Fuzzy logic reasoning based approach for the Environmentally Responsibility assessment. The modeling has been performed using Matlab Fuzzy Logic Toolbox. Comparison between BRB and Fuzzy approach results has been performed and proved the validity of a proposed BRB technique.

In the last two decades the fuzzy logic theory application have increased significantly, which was firstly introduced by L.A. Zadeh [13]. Fuzzy logic (FL) comes from theory of fuzzy sets, where classes of input objects have unsharp boundaries with a certain degree of belief, which is in a wider sense can be described as a theory of multivalued logic. FL has been widely deployed in different applications of decision support, industrial process controls and consumer products selection. A crucial significance of that approach is the use of linguistic variables in describing complex systems, e.g. descriptive words in human-readable and comprehensible format [14].

\section{A. Fuzzy Logic design}

A basic fuzzy logic system consists of three component: fuzzifier, FIS and defuzzifier. A fuzzifier finds a mapping between input variables values into a fuzzy set and defuzzifier does a reverse operation of mapping set of output values into a crisp value. Hence, fuzzy logic is a helpful tool to map an input space to an output space. The primary mechanism for doing this resides in FIS through the list of if-then rules. An architectural view of basic fuzzy system is shown in Fig. 6: 


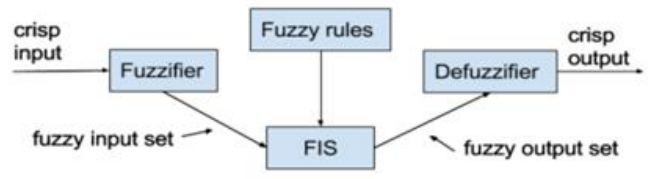

Fig. 6. Fuzzy logic system design

There are three steps in a fuzzy inference process: fuzzification, fuzzy rule inference, and defuzzification. The algorithmic steps of a fuzzy logic system life cycle are as follows:

1. Decide on linguistic variables and notations for input and output variables.

2. Determine pertinent membership functions for each input and output variables.

3. Construct knowledge base in rules format.

4. Fuzzification: convert crisp data into fuzzy data sets using membership functions.

5. Fuzzy rules inference: rules evaluation in the rule base and the results set combination.

6. Defuzzification: convert fuzzy output values into crisp data.

The Mamdani type inference has been deployed for building the model, which expects the output membership functions to be fuzzy sets. The input variables are fuzzified with three linguistic attributes "low", "medium" and "high", as it was defined in a BRB system earlier, with the gaussmf membership function (MF) in a range [0 100]. The output variable is described with trimf MF with "poor", "average" and "good" attributes. The model consists of 3 rules, defined in accordance with the BRB system described earlier. The Surface Viewer of the Matlab Fuzzy Logic Toolbox shows the graphical mapping between any two inputs and an output.

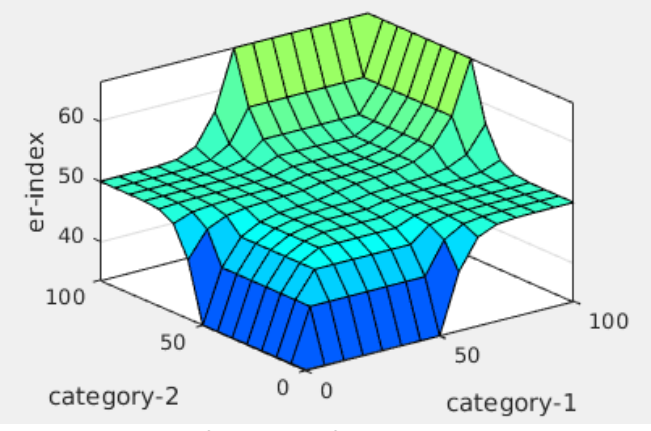

Fig. 7. Surface view of ER SME assessment

\section{B. Results and comparison}

Belief Rule-Based approach is compared with FIS in this section. In order to verify the validity of the methodology chosen in ER assessment research, 100 simulations with randomized input variables (answers to questions) were carried out. The chart below presents results of simulations, an additional mean value is included in comparative analysis as a benchmarking number.

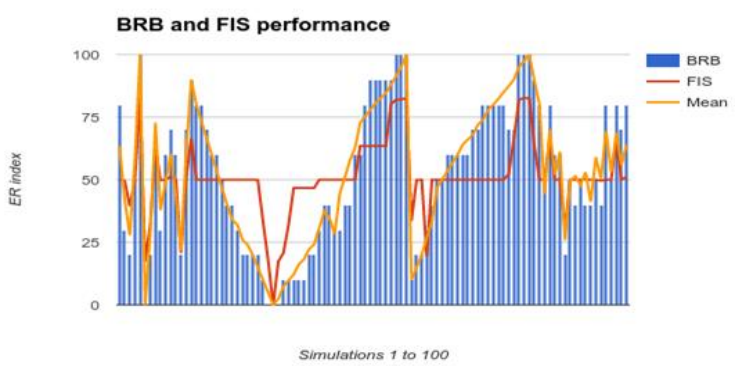

Fig. 8. Methods comparison 
It is observed that the results of fuzzy approach are close to the results obtained by the average calculation method for almost all the experiments. All three methods (third is the average value calculation) results are discovered in the same range of $[-20:+20]$ and FIS performs better for the range of values closer to medium, not reaching min or max with the deviation value. Per category effect and consequence are not handled in output variables generated based on mean value calculations. For example, when the "Category 1 - Equipment procurement compliant with Green ICT guidelines and the optimization of enterprise operations" accomplishes its max value, that implies a positive degree of awareness of an organization on environmental issues, hence result in BRB in simulation1 is higher compared to FIS and mean calculations. Also, when at least one of the input variables is equal to zero, BRB approach demonstrates a lesser output variable, which is legitimate: failing to address even one aspect of a problem, causes the whole case status unsteady and unreliable.

\section{Statistical Analysis and Significance Tests}

The significance test has been performed to determine if the BRB approach and FIS theory produce significantly different results. As a first step, we need to test the assumption that the samples come from normal distributions. From the plots below it is evident that BRB (blue) approximately follows straight line, indicating approximate normal distribution. The FIS sample (orange colored) shows an increasing departure from normality in the lower tail.

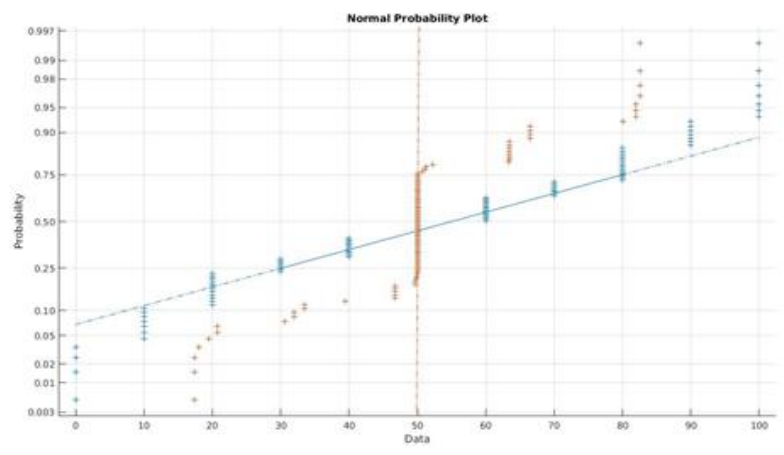

Fig. 9. Normal distribution probability for BRB and FIS

The difference between BRB and FIS is evident. This can be confirmed performing Jarque-Bera test in Matlab. The returned value of $h=1$ for FIS indicates that jbtest rejects the null hypothesis at the default $5 \%$ significance level. The logical 0 returned by BRB test indicates a failure to reject the null hypothesis that the samples are normally distributed.

Next, we need to set up hypotheses and evaluate whether the difference between BRB and FIS is significant. In this statistical test, $\mu 1$ is the mean score for BRB while $\mu 2$ is the mean score for FIS. HO is the null hypothesis where $\mu 1$ equals to $\mu 2$ while $\mathrm{H} 1$ is the alternative hypothesis where $\mu 1$ is not equal to $\mu 2$. A two-tail z-test is conducted at a level of confidence of 0.05 . difference between BRB and FIS is evident. This can be confirmed performing JarqueBera test in Matlab. The returned value of $\mathrm{h}=1$ for FIS indicates that jbtest rejects the null hypothesis at the default $5 \%$ significance level. The logical 0 returned by BRB test indicates a failure to reject the null hypothesis that the samples are normally distributed.

$$
\mathrm{H} 0: \mu 1=\mu 2 \mathrm{H} 1: \mu 1 \neq \mu 2, \alpha=0.05
$$

The Table 3 below shows a statistical analysis of the two independent data samples. Because both data samples are large (>30), a z-test has been chosen opposed to t-test. Prior to conducting the z-test, we first calculate the population variances. The variance values are different for BRB (819.43) and FIS (194.70) and thus we shall conduct a two-tail z-test for two samples with unequal variances at a level of confidence of 0.05 .

TABLE 3 - z-Test: two Sample for Means

\begin{tabular}{|l|l|l|}
\hline & $B R B$ & $F I S$ \\
\hline Mean, $\mu$ & 52.32 & 51.01 \\
\hline Known Variance, $\sigma$ & 819.43 & 194.70 \\
\hline No of observations $(\mathrm{n})$ & 99.00 & 99.00 \\
\hline
\end{tabular}




\begin{tabular}{|l|l|l|}
\hline Hypothesized Mean Difference & 0.00 \\
\hline$Z$ value & 0.41 & \\
\hline$P(Z<=z)$ one-tail & 0.34 & \\
\hline z Critical one-tail & 1.64 & \\
\hline$p$-value ( $<<=z)$ two-tail & 0.68 & \\
\hline z Critical two-tail & 1.96 & \\
\hline
\end{tabular}

The $p$-value is a probability that measures the evidence against the null hypothesis. The $p$-value in our research is greater than $\alpha$, so we fail to reject HO. This means that BRB and FIS approaches do not generate significantly different results. However, as we can see from the Table 9, the variances of two populations are unequal (BRB is 4 times higher than FIS). Again, the hypotheses-test indicates that there is not enough evidence to reject the null hypothesis that the two batch z-score is equal at the 0.05 significance level. This failure may reflect normality in the population or it may reflect a lack of strong evidence against the null hypothesis due to the small sample size.

A two-tail z-test for the means of two independent samples with unequal variances investigates whether two independent samples come from normal distributions with unequal variances and the same mean values. From Jarque-Bera test it has been identified that FIS data sample is not normally distributed. For a hypotheses test quality assurance of two data samples have been tested also using the two-sample Kolmogorov-Smirnov test. The function kstest2(columnBRB, columnFIS) returned the value equal to 1 . The result rejects the hypothesis that the data in BRB and FIS samples are from the same continuous distribution at the $5 \%$ significance level. This confirms our assumption in two approaches heterogeneity.

To conclude, the BRB approach deployed in this research dedicated to small and medium-sized enterprises to assess their Environmentally Responsibility level generates correlative, well balanced and sensible results compared to FIS approach. So the observed difference between the sample means $(52.32-51.01)$ is not convincing enough to say that BRB and FIS approaches generate notably different consequences. Although, the test only performs comparison between mean values and does not focus on such a substantial difference in variances. The most likely explanation of z-test result is that BRB approach deploys rule based context-adapted inference procedures for a total consequent variables calculation.

\section{RESEARCH RESULTS AND DISCUSSION}

For the purpose of ER BRB assessment validation, evaluation sessions have been held with experts, non-experts, and the target SMEs. Six domain experts were invited to perform formative and summative evaluations to address validity, reliability and accuracy of a system developed:

1. British Library employee, graduated from Leeds Beckett University majoring in ICT for Environment;

2. Web Operations Manager/Technical Director;

3. Professor at University of Hull and BCS Green IT Specialist Group member;

4. Carbon3IT Ltd Sustainable IT consultancy Managing Director, BCS Green IT Specialist Group member;

5. Senior Lecturer, Course Leader for MSc Sustainable Computing, School of Computing, Creative Technologies and Engineering, Faculty of Arts, Environment and Technology at Leeds Beckett University.

6. Head of School of Computing, Creative Technologies and Engineering, Faculty of Arts, Environment and Technology at Leeds Beckett University.

Summing up the results, participants expressed positive opinions about the system, reviewed that the software has been developed in compliance with specifications, software systems development standards and best practices.

Environmentally responsibility problems are represented with a large number of non-exhaustive factors. The literature review revealed inconsistency and diversity in existing assessment models which indicates the domain haven't reached its saturation point and is not conclusive. Clearly, further research is required in Green ICT readiness domain categorization dedicated to enterprises.

The originality of solution lies in the deployment of Belief Rule-Based approach in assessment of such a broad concept as Environmentally Responsibility. Another important implication of these findings is that the Recommendations part of BRB system will help enterprises to focus on making improvements on operating in a more sustainable way. 


\section{CONCLUSION}

This paper shows that BRB methodology gives more leverage in inference procedures compared to traditional summative methods used in existing models analyzed in Literature Review. BRB technique could be applied in other problems with uncertain, ambiguous and fuzzy knowledge-based systems. Statistical significance analysis results clearly show the validity of a Belief Rule-Based approach correlated with Fuzzy inference system. By defining each possible combination of input attributes in rules and executing corresponding inference procedures the BRB system is believed to generate well balanced, sensible and context adapted results.

Future work recommendations are drawn from the validation by experts, focus groups, and the target SM, which are: 1 ) test the usefulness of the assessment in practice and benchmark the results among a pool of similar type of enterprise respondents; 2) include required capital investment amount, payback period and cost saving for each recommended activity; 3) provide specialized assessment and recommendation roadmaps for a participating organization's industrial sector (manufacturing, non-manufacturing).

The key limitations of this study are: 1) assessment does not take into account regional differences among potential participating organizations; 2 ) assessment model is not sector-specific. The final limitation extracted from validation sessions is the assessment model applicability to medium-sized enterprises, rather than small and micro organizations. This is due to the type of content and level of questions asked in the assessment process.

\section{ACKNOWLEDGMENT}

This work has been conducted as part of the Erasmus+ Master program PERCCOM (Pervasive Computing and Communications for Sustainable Development) [15]. Thus, author would like to thank the Erasmus Mundus program and the European Union for the opportunity to participate in this program and complete this study.

The authors are grateful to referees for their feedback comments that have helped to improve the quality of the system.

\section{RefERENCES}

[1] A. Crane, D. Matten and L. J. Spence, "Corporate social responsibility in a global context," Chapter in: Crane, A., Matten, D., and Spence, $\amalg$,'Corporate Social Responsibility: Readings and Cases in a Global Context, vol. 2, pp. 3-26, 2013.

[2] The Climate Group, "SMART 2020: Enabling the low carbon economy in the information age," 2008.

[3] A. Martinuzzi, R. Kudlak, C. Faber and A. Wiman, "CSR activities and impacts of the retail sector," 2011.

[4] A. Hankel, L. Oud, M. Saan and P. Lago, "A maturity model for green ICT: The case of the SURF green ICT maturity model." in Enviroinfo, 2014, pp. 33-40.

[5] Sustainability Maturity Model from Industrial Research Institute (IRI).

[6] Fachgruppe Green IT Action Plan model.

[7] UK HM Government Green ICT Maturity Model.

[8] A. Molla, V. Cooper and S. Pittayachawan, "The Green IT readiness (G-Readiness) of organizations: An exploratory analysis of a construct and instrument," Communications of the Association for Information Systems, vol. 29, pp. 67-96, 2011.

[9] S. deMonsabert, K. Odeh and J. Meszaros, "SustainaBits: A framework and rating system for sustainable IT," in Green Computing Conference (IGCC), 2012 International, 2012, pp. 1-9.

[10] Jian-Bo Yang, Jun Liu, Jin Wang, How-Sing Sii and Hong-Wei Wang, "Belief rule-base inference methodology using the evidential reasoning Approach-RIMER," IEEE Transactions on Systems, Man, and Cybernetics - Part A: Systems and Humans, vol. 36, pp. 266-285, 2006.

[11] Y. Chen, J. Yang, D. Xu, Z. Zhou and D. Tang, "Inference analysis and adaptive training for belief rule based systems," Expert Syst. Appl., vol. 38, pp. 12845-12860, 2011.

[12] European Commission, Joint Research Centre, "Best environmental management practice in the telecommunications and ICT services sector," Institute for Prospective Technological Studies, 2015.

[13] L. A. Zadeh, "Toward a theory of fuzzy information granulation and its centrality in human reasoning and fuzzy logic," Fuzzy Sets Syst., vol. 90, pp. 111-127, 1997.

[14] L. A. Zadeh, "Fuzzy sets," Information and Control, vol. 8, pp. 338-353, 1965.

[15] A. Klimova, E. Rondeau, K. Andersson, J. Porras, A. Rybin, A. Zaslavsky, "An international Master's program in green ICT as a contribution to sustainable development", Journal of Cleaner Production, 2016. 